\title{
A semi-empirical model for streamwise vortex intensification
}

\author{
Dr. Grant McLelland ${ }^{1}$ \\ Centre for Propulsion \\ School of Aerospace, Transport and Manufacturing \\ Cranfield University \\ Bedford, MK43 0AL, UK \\ Email: g.s.mclelland@cranfield.ac.uk \\ Phone: +44(0) 1234754463 \\ Prof. David MacManus \\ Centre for Propulsion \\ School of Aerospace, Transport and Manufacturing \\ Cranfield University \\ Bedford, MK43 0AL, UK \\ Email: $\underline{\text { d.g.macmanus@cranfield.ac.uk }}$ \\ Mr. Chris Sheaf \\ Intake Aerodynamics Specialist, Nacelle Engineering \\ Rolls Royce, Derby, DE22 8BJ, UK \\ Email: chris.sheaf@rolls-royce.com
}

\begin{abstract}
Vortex intensification plays an important role in a wide range of flows of engineering interest. One scenario of interest is when a streamwise vortex passes through the contracting streamtube of an aircraft intake. There is, however, limited experimental data of flows of this type to reveal the dominant flow physics and to guide the development of vortex models. To this end, the evolution of wing-tip vortices inside a range of streamtube contractions has been measured using Stereoscopic Particle Image Velocimetry. A semi-empirical model has been applied to provide new insight on the role of vorticity diffusion during the intensification process. The analysis demonstrates that for mild flow contractions, vorticity diffusion has a negligible influence due to the low rates of diffusion in the vortex flow prior to intensification and the short convective times associated with the streamtube contraction. As the contraction levels increase, there is a substantial increase in the rates of diffusion which is driven by the greater levels of

${ }^{1}$ Corresponding author
\end{abstract}


vorticity in the vortex core. A new semi-empirical relationship, as a function of the local streamtube contraction levels and vortex Reynolds number, has been developed. The model comprises a simple correction to vortex filament theory and provides a significant improvement in the estimation of vortex characteristics in contracting flows. For the range of contractions investigated, errors in the estimation of vortex core radius, peak tangential velocity and vorticity are reduced by an order of magnitude. The model can be applied to estimate the change in vortex characteristics for a range of flows with intense axial strain, such as contracting intake streamtubes and swirling flows in turbomachinery.

\section{Keywords}

Vortex, vortex intensification, vortex modelling, Particle Image Velocimetry, PIV, intake flows

\section{Introduction}

Future airframe configurations are likely to feature an increased aerodynamic coupling between the intake and the airframe ${ }^{1}$. For example, the rear-mounted engines of blended wing body configurations may be susceptible of the ingestion of discrete streamwise vorticity which has been generated upstream on the airframe at high airframe angles of attack $^{2}$. The risk is also present on conventional fuselage arrangements due to vortices from the wing-body junction or at the wing-tip of a forebody canard. This is of concern from an engine integration perspective since streamwise vortices can result in high-cycle fatigue on compressor blades ${ }^{3}$, as well as a reduction in surge margin ${ }^{4,5}$. The extent of the intake flow distortion, as well as the peak flow angles, are known to be a critical parameter for the response of the turbomachinery to the distorted flow ${ }^{1}$. Therefore, it is useful to determine the nature of such vortical flows once they have been ingested by the intake. It is envisaged that the greatest risk of vortex ingestion occurs during take-off and climb-out when the diameter of the intake capture streamtube is relatively large and the airframe angle of attack is increased. In these conditions, the vortex will be subject to axial strain due to the contracting intake streamtube. Fundamental theory shows that the vortex will undergo intensification in response to the flow contraction ${ }^{6}$. The inverse occurs when the streamtube is diffusive in nature, and vortex breakdown may occur ${ }^{7}$. Vortex intensification plays an important role in a range of other flows of engineering interest, such as helicopter wake intensification during flight close to ground ${ }^{8-10}$, vortexpropeller interactions ${ }^{11}$, drain-hole flows ${ }^{12}$, swirling flow intensification in the inlet guide vanes for turbines in gas turbine engines ${ }^{13,14}$, and the evolution of the vortex flows in the secondary flow of turbines ${ }^{15,16}$. 
Vortex filament theory demonstrates that a streamtube contraction results in a vortex with a reduced core radius, in conjunction with an increase in streamwise vorticity and tangential velocity ${ }^{17}$. Analytical solutions to the inviscid, axisymmetric Navier-Stokes Equations for confined swirling flows were developed by Batchelor ${ }^{18}$. Numerical solutions have also been studied for a range of swirling flows inside a confined contraction or diffusion ${ }^{19-22}$, with a particular focus on the susceptibility of the flow to vortex breakdown. The dominant flow physics are driven by the constraints enforced by the bounding surfaces of the pipe, and there exist solutions in which separated flow can form on the walls ${ }^{19}$. Burgers ${ }^{23}$ and Rott ${ }^{24}$ developed solutions to the axisymmetric Navier-Stokes Equations for the case of a vortex in a uniform streamwise velocity gradient, such as that which is experienced in drain-hole flows ${ }^{12}$. The solutions are for asymptotically-steady flow in which the intensification of vorticity due to the streamwise contraction is balanced by viscous diffusion.

There have, however, been limited experimental studies to guide the understanding of the evolution of streamwise vortices in flow contractions such as those of interest for intake flows. Garbeff et al. ${ }^{25}$ acquired three-component hot wire measurements of a wing tip vortex inside a mild planar contraction of velocity ratio $\left(w / w_{0}\right) 1.4$, where $w$ is the streamwise velocity and subscript 0 refers to the initial condition at the start of the contraction. The measurements demonstrated that the peak tangential velocity increased by $10 \%$ relative to the value at the start of the contraction, although there was no overall change in core radius. The distribution of streamwise velocity inside the contraction, as well as the initial circulation distribution of the vortex, were used as inputs for the Rott vortex model. The model estimated an increase in peak tangential velocity of $6 \%$, which is somewhat lower than the measured increase of $10 \%$. The authors cited the relatively low spatial resolution of the measurements as a contribution to differences between the model and the experiments. Additionally, it is widely known that point-based measurements of streamwise vortices can be strongly impacted by wandering, and must be corrected ${ }^{26,27}$. As the results of Garbeff et al. ${ }^{25}$ were not corrected for wandering, additional uncertainty is introduced in the comparison between the experimental measurements and the results from the Rott model. This issue can be mitigated more easily using synchronous planar measurements, such as those obtained using Particle Image Velocimetry (PIV).

Ananthan and Leishman ${ }^{8}$ and Ramasamy and Leishman ${ }^{9}$ used Laser Doppler Velocimetry (LDV) to measure a helicopter rotor tip vortex for the case where the rotor is located in close proximity to a ground plane. As the rotor wake convects towards the ground, the velocity field stretches the wake in the radial direction. This flow field results in a corresponding intensification of the tip vortex, and a reduction in core radius of approximately $38 \%$ was measured. A model for the evolution of the tip vortex was 
developed, comprising vortex filament theory to capture the strain effects, coupled with the Squire vortex model ${ }^{28}$ to account for the effects of vorticity diffusion. Using this approach the overall trend in the change of vortex core radius throughout the wake evolution was captured adequately. However, only the predictions of core radius from the model were compared with the measurements. As for Garbeff et al. ${ }^{25}$, there was no direct comparison with vortex filament theory, so the role of diffusion was not quantified. In addition, the analysis was performed for only one particular vortex Reynolds number and flow field. Therefore, it was not possible to elucidate the sensitivity of the intensification process to these parameters.

McLelland et al. ${ }^{29}$ performed a detailed parametric study on the evolution of a streamwise vortex within a streamtube contraction. A sub-scale intake was employed to generate streamtubes of velocity ratios between $w_{i} / w_{0}, 2.0$ and 16.3, where $w_{i}$ is the mean velocity at the throat of the intake and $w_{0}$ is the velocity in the freestream flow. The vortex Reynolds number was controlled by independent variation of $w_{0}$ and the vortex generator angle of attack, $\alpha_{v g}$. The measurements provided the first controlled parametric assessment on the intensification of vortices, and the first direct comparison between vortex filament theory and measured vortex intensification. It was demonstrated that the vortex intensification process is in good agreement with vortex filament theory when the local streamtube contraction levels are relatively low, until approximately $w / w_{0}=2.0$. At greater levels of flow contraction, the vortex experienced less intensification than anticipated. At the greatest level of streamtube contraction which was investigated $\left(w / w_{0}\right.$ $=6.5)$ the core radius $\left(r_{c}\right)$ of the measured vortex was $27 \%$ greater than that which was expected from vortex filament theory, with a peak vorticity $\left(\omega_{z, \max }\right)$ of $119 \%$ lower. The behaviour was found to be consistent with vorticity diffusion, the impact of which became increasingly important as the local contraction levels were increased. The evolution of the peak vorticity was found to be sensitive to the vortex Reynolds number $\left(R e_{v}\right)$, such that the change in peak streamwise vorticity was smaller as $R e_{v}$ is increased. Overall, the study underlined that the evolution of the vortex characteristics is captured poorly by fundamental theory for large contraction levels, and the effects of diffusion must be modelled.

This research presents an approach to quantify the contribution of vorticity diffusion to the vortex intensification process. Detailed Stereo PIV measurements from the controlled parametric studies in McLelland et al. ${ }^{29}$ are employed. First the Experimental Methods and Approach section provides a summary of the experimental arrangement, the measurement methods, the measurement uncertainties and the test matrix. The Model Development section provides details on the elements of the semi-empirical model which is used in the present study. In the Results section, new insight into the role of vorticity diffusion is obtained from the development of a new relationship for the semi-empirical 
model. Finally, the performance of the new model is assessed with the evaluation of the predicted and measured vortex characteristics across a range of streamtube contractions and vortex characteristics.

\section{Experimental Methods and Approach}

\subsection{Experimental arrangement}

The experiments were performed in the low-speed wind tunnel at Cranfield University. The tunnel features a working section of dimensions $2.4 \times 1.8 \mathrm{~m}$, and can attain a maximum freestream velocity of approximately $40 \mathrm{~ms}^{-1}$. A sub-scale intake model of inner diameter $D_{i}=0.1 \mathrm{~m}$ was mounted at the centre of the working section and was aligned with the freestream direction (Figure 1). Flow through the intake model was established by means of a suction system which comprised a vacuum tank of capacity $60 \mathrm{~m}^{3}$ and a slide valve to control the mass flow through the intake. It was thus possible to establish streamtube contractions of various intensities as characterised by the intake velocity ratio $V R=w_{i} / w_{0}$, where $w_{i}$ is the velocity inside the intake and $w_{0}$ is the freestream velocity. Steady flow through the intake was maintained for a minimum of 20 $\mathrm{s}$ at the maximum mass flow rate of $1.47 \mathrm{kgs}^{-1}$.

The freestream velocity wo was measured using a Pitot-static probe which was mounted in the working section. A Furness Controls FC-044 pressure transducer with a total range of $2.5 \mathrm{kPa}$ was employed to measure the Pitot-static pressures. The flow inside the intake was determined using measurements from four static pressure ports which were located on the inner surface of the intake at a distance of $0.65 D_{i}$ downstream of the highlight plane. The pneumatic average of the static pressure ports was measured using an Omega PX138-005D4V differential pressure transducer of maximum range $34.4 \mathrm{kPa}$. The static pressure inside the intake was used in conjunction with the freestream total temperature to calculate the isentropic Mach number inside the intake. Therefore, it was possible to determine the velocity $w_{i}$ and so the intake velocity ratio $V R$. The pressure data was acquired using a National Instruments PCI-6255 16-bit digital acquisition card at a rate of $600 \mathrm{~Hz}$ and for a duration of $5 \mathrm{~s}$ during each run.

The measurement uncertainties for the transducers considered contributions from the transducer accuracy, the resolution error and the calibration error. It was determined from quadrature that the overall uncertainties on the freestream velocity and the intake flow velocity were $1.0 \%$ and $3.2 \%$ of typical values. This resulted in an uncertainty on the intake $V R$ of $3.4 \%$. 


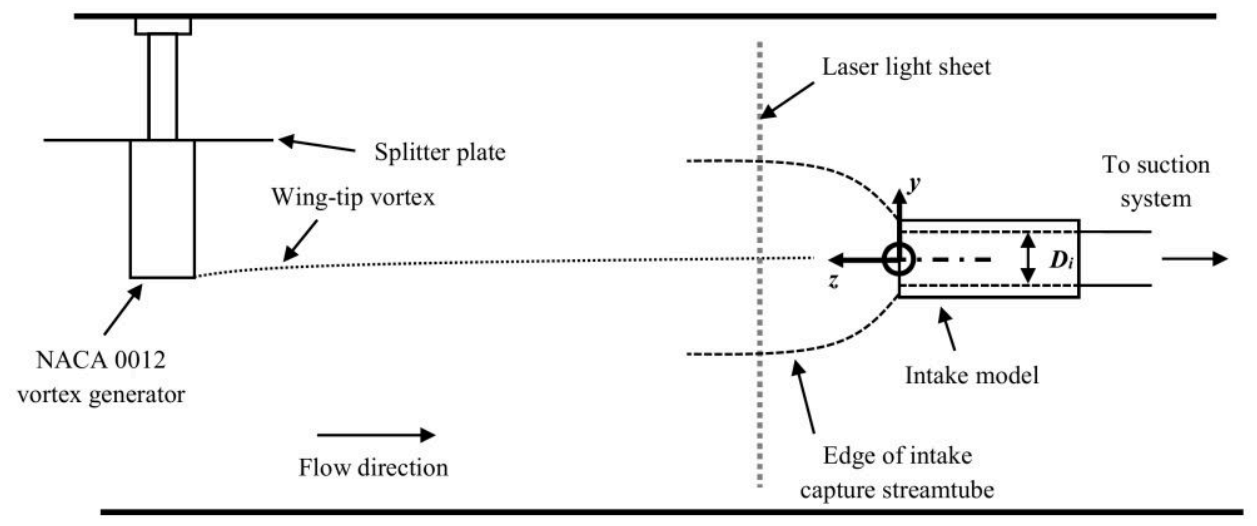

Figure 1: Schematic of wind tunnel arrangement, side view

A single streamwise vortex was generated by means of a vortex generator of NACA 0012 section which was mounted in the working section and upstream of the intake. The vortex generator had a chord length of $0.15 \mathrm{~m}$ and a semi-span of $0.45 \mathrm{~m}$. The position of the vortex generator was adjusted during the experiment to ensure that the tip vortex was located at the centre of the streamtube contraction. For all configurations, the timeaveraged centre of the vortex was found to be located within $0.05 D_{i}$ of the intake centreline. Further details of the experimental arrangement are reported in McLelland et al. ${ }^{29}$.

\subsection{S-PIV measurements}

Stereoscopic Particle Image Velocimetry (S-PIV) was employed to measure the threecomponent velocity field on planes which were perpendicular to the freestream velocity direction. The laser light was generated using a $200 \mathrm{~mJ}$ dual-pulsed Nd:YAG laser with a wavelength of $532 \mathrm{~nm}$ and a maximum frequency of $15 \mathrm{~Hz}$. The laser beam was transformed into a light sheet of thickness $1.5 \mathrm{~mm}$ with use of plano-concave and planocylindrical lenses. Two cameras of resolution $4 \mathrm{MPx}$ were utilised with $105 \mathrm{~mm}$ focal length lenses to image the region of interest. The cameras were placed upstream of the intake and on the horizontal plane ( $y=0 \mathrm{~mm}$, Figure 1). Each camera was located with a rotation angle of $45^{\circ}$ between the lens centreline axis and the tunnel centreline, and Scheimpflug mounts were employed to ensure focus across the region of interest. The optical setup resulted in a region of interest of width $160 \mathrm{~mm}$ and of height $100 \mathrm{~mm}$. A total of 200 velocity field measurements were obtained at a rate of $7.5 \mathrm{~Hz}$.

All images were processed using a linear deformation grid algorithm with a 50\% interrogation window overlap. Multi-pass processing was employed with an initial window size of 64 px and a final size of 32 px. Invalid vectors were identified using a maximum displacement tolerance of $16 \mathrm{px}$ and a signal to noise ratio (SNR) threshold of 1.2. Additionally, local vector validation was performed using the universal median test 
${ }^{30}$ with a non-dimensional tolerance of 3 applied on a stencil size of $3 \times 3$ vectors. The invalid vectors were subsequently replaced using the local mean of vectors on a $3 \times 3$ stencil. The processing strategy resulted in a vector spatial resolution of approximately $0.01 D_{i}$, and typically less than $2 \%$ of the vectors in the region of interest were deemed invalid.

For each instantaneous measurement, the position of the vortex centre was identified using the peak streamwise vorticity and the velocity fields were re-aligned to the position of the centre of the vortex. Subsequently, The vortex characteristics were determined using the Vorticity Disk Method ${ }^{31}$. In this approach, a circular grid of maximum radius $r_{\max } / D_{i}=0.25$ was placed at the centre of the vortex. The circular grid had a resolution of 150 points in the radial direction and 261 points in the circumferential direction to resolve the vorticity distribution sufficiently. Linear interpolation was used to interpolate the streamwise vorticity from the PIV measurement grid to the circular grid. The streamwise vorticity was integrated on each annulus with increasing radius which results in the circumferentially-averaged distribution of circulation, $\Gamma(r)$. It is then possible to derive the circumferentially-averaged tangential velocity distribution, $V_{\theta}(r)$ with use of Eq.1. Finally, using the profiles of $V \theta(r)$ and $\Gamma(r)$ the instantaneous values of core radius, peak tangential velocity, and core circulation were obtained, after which average velocity field was computed. A key benefit of this this approach is that it avoids the artificial diffusion of the vortex characteristics which typically arises due to vortex wandering ${ }^{26,27}$.

$$
V_{\theta}(\mathrm{r})=\frac{\Gamma(r)}{2 \pi r}
$$

The uncertainties in the PIV velocity vectors were estimated using the analysis of Raffel et al. ${ }^{32}$. The estimate includes contributions from the particle image displacement, particle image diameter, seeding density, image quantisation level, and background noise. An estimate of the disparity error due to misalignment between the camera calibration plane and the light sheet has also been included in the uncertainty analysis. Based on the preceding assessment, the uncertainty in the peak tangential $\left(\delta V_{\theta} / V_{\theta, 0}\right)$ and out of plane velocities $\left(\delta w / w_{0}\right)$ were found to be $9 \%$ and $4 \%$ of typical values, respectively. This results in an uncertainty in peak vorticity $\left(\delta \omega_{z} / \omega_{z, 0}\right)$ of $18 \%$, and a corresponding uncertainty on the circulation $\left(\delta \Gamma / \Gamma_{0}\right)$ of $8 \%$. The uncertainty on the vortex core radius $\left(\delta r_{c} / r_{c, 0}\right)$ is dictated by the final spatial resolution of the PIV velocity vectors and corresponds to $7 \%$. 


\subsection{Test matrix}

Measurement plane positions of $z / D_{i}=[2.25,0.8,0.6,0.38,0.20]$ were investigated to capture the evolution of the vortex as it moves through the streamtube contraction. The position of the measurement plane was fixed at a distance of $z / c=6.0$ downstream of the trailing edge of the vortex generator, and the intake highlight plane was translated in the $z$-direction to reduce the complexity of the experimental arrangement ${ }^{29}$. Based on estimates using the Squire vortex model ${ }^{28}$, the change in the vortex characteristics over the distance that the intake was translated is expected to be within the experimental uncertainty ${ }^{29}$. Measurements were also acquired with the intake removed from the working section to determine the vortex characteristics in the unperturbed freestream flow.

Two key variables are of interest in this study. The first corresponds to the streamtube contraction levels, which have been characterised in terms of the intake velocity ratio, $\mathrm{VR}=w_{i} / w_{0}$. A range of streamtube contraction levels were assessed through the control of the intake mass flow at a particular freestream velocity. The maximum $V R$ was 5.2 at $w_{0}=35.4 \mathrm{~ms}^{-1}$ and 16.3 at $w_{0}=11.0 \mathrm{~ms}^{-1}$. The minimum $V R$ investigated was $V R=2.0$ at $\mathrm{w}_{0}=17.2 \mathrm{~ms}^{-1}$. The second variable of interest in this study is the vortex Reynolds number $\operatorname{Re}_{v}=\Gamma_{c} d v$, where $\Gamma_{c}$ is the core circulation of the vortex and $v$ is the kinematic viscosity. Previous correlations of wing-tip vortex measurements have demonstrated that the rate of vortex diffusion, as identified from the evolution of the vortex core radius in time, is a function of $\operatorname{Re}_{\mathrm{v}}{ }^{33,34}$. For $\mathrm{Re}_{\mathrm{v}}$ of up to $10^{3}$, the rate of diffusion is approximately equal to the rate which is anticipated under the action of laminar viscosity. At greater values of $\mathrm{Re}_{\mathrm{v}}$ the rate of diffusion increases, which is typically attributed to a rise in turbulent diffusion in the vortex core ${ }^{33}$. Two approaches have been utilised to provide a change in the vortex Reynolds number. The first was to vary the freestream velocity ( $w_{0}$ $=11.0,17.2,35.4 \mathrm{~ms}^{-1}$ ) for a fixed angle of attack of $\alpha_{v g}=12^{\circ}$. An increase in $w_{0}$ produces an increase in the total circulation of the wing, with a resultant increase in the vortex Reynolds number of the wing-tip vortex. Vortex Reynolds numbers of $2.4 \times 10^{4}$, $3.7 \times 10^{4}$ and $7.4 \times 10^{4}$ were achieved at an intake $V R$ of approximately 5.0. The second means to vary $R e_{v}$ was to reduce the lift coefficient of the wing through the reduction of the angle of attack. A single condition was investigated for $\alpha_{v g}=6^{\circ}$ at $w_{0}=35.4 \mathrm{~ms}^{-1}$, which resulted in $R e_{v}=2.3 \times 10^{4}$. 
Table 1: Experimental conditions and wing-tip vortex characteristics, NACA 0012 vortex generator, vortices ingested at the intake centreline

\begin{tabular}{llllll}
\hline$\alpha_{V G}$ [deg] & $R e_{c}=w_{0} / v$ & $R e_{v}=\Gamma_{c} / v$ & $r_{c}^{*}=r_{c, 0} / D_{i}$ & $\omega_{z, a v}{ }^{*}=\omega_{z, a v, 0} D_{i} / w_{\text {ref }}$ & $V R=w_{i} / w_{0}$ \\
\hline 12 & $1.1 \times 10^{5}$ & $2.4 \times 10^{4}$ & 0.092 & 3.75 & 5.2 \\
12 & $1.1 \times 10^{5}$ & $2.4 \times 10^{4}$ & 0.092 & 3.75 & 16.3 \\
12 & $1.7 \times 10^{5}$ & $3.7 \times 10^{4}$ & 0.087 & 6.52 & 2.0 \\
12 & $1.7 \times 10^{5}$ & $3.7 \times 10^{4}$ & 0.087 & 6.52 & 5.1 \\
12 & $1.7 \times 10^{5}$ & $3.7 \times 10^{4}$ & 0.087 & 6.52 & 10.3 \\
12 & $3.6 \times 10^{5}$ & $7.4 \times 10^{4}$ & 0.082 & 14.66 & 4.9 \\
6 & $3.6 \times 10^{5}$ & $3.4 \times 10^{4}$ & 0.065 & 10.93 & 4.9 \\
\hline
\end{tabular}

\section{Model development}

The detailed measurements in McLelland et al. ${ }^{29}$ demonstrated that the overall effect of vorticity diffusion becomes increasingly important as the local contraction levels $\left(w_{c} / w_{c, 0}\right)$ increase and, as a result, a simple inviscid relationship becomes less suitable. It is desirable to characterise the influence of diffusion, and to determine if the diffusion rates are consistent with those which are expected for vortices of this range of vortex Reynolds numbers. To do this, an appropriate model for vorticity diffusion can be developed to account for the differences between the measurements and vortex filament theory. Consider a streamwise vortex with an initial core radius of $r_{c, 0}$ measured upstream of the streamtube contraction at position $O$ with a streamwise velocity of $w_{c, 0}$ at the vortex centre (Error! Reference source not found.). Inside the contraction, the streamwise velocity has increased to $w_{c}$ and the core radius has reduced to $r_{c}$. As a first approximation, $r_{c}$ can be considered to be composed of an inviscid contribution $\Delta r_{c, V F}$ due to intensification, plus an additional component $\Delta r_{c, D}$ due to vorticity diffusion (Eq. 2). This approach is consistent with the one utilised by Karpatne et al. ${ }^{10}$ to model rotor wake intensification. The inviscid contribution $\Delta r_{c, V F}$ is calculated from vortex filament theory and can be expressed as Eq. 3. For a streamtube contraction, $\Delta r_{c, V F}$ is negative, and viscous diffusion results in a positive value of $\Delta \mathrm{r}_{\mathrm{c}, \mathrm{D}}$. 


$$
\begin{aligned}
& r_{c}=r_{c, 0}+\Delta r_{c, V F}+\Delta r_{c, D} \\
& \Delta r_{c, V F}=r_{c, 0}\left[\sqrt{\frac{w_{c, 0}}{w_{c}}}-1\right]
\end{aligned}
$$

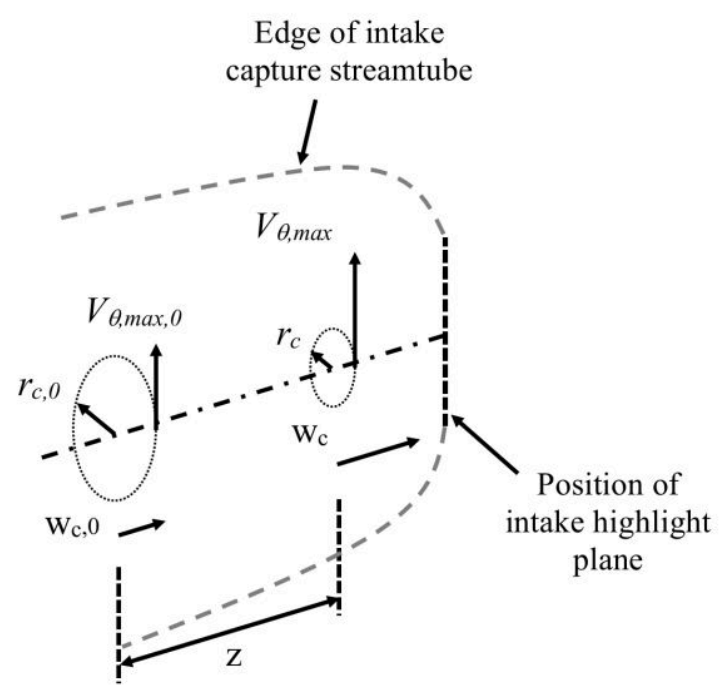

Figure 2: Schematic of intake flow field and definitions for vortex intensification model

\subsection{Vortex filament theory}

Vortex filament (VF) theory can be utilised to determine how closely the measured levels of vortex intensification follow fundamental inviscid, incompressible vortex dynamics. From the second Helmholtz law of vortex motion, a vortex filament moves with the fluid ${ }^{6}$. Therefore, the filament undergoes a reduction in cross-sectional area which is proportional to the increase in streamwise velocity $\left(w_{c} / w_{c}\right.$, ), as dictated by continuity. Assuming that the core radius $\left(r_{c}\right)$ defines the edge of the vortex filament, then the change in vortex core radius relative to that in the unperturbed flow $\left(r_{c} / r_{c, 0}\right)$ can be expressed as a function of $w_{c} / w_{c, 0}$ (Eq. 4).

$$
\frac{r_{c}}{r_{c, 0}}=\sqrt{\frac{w}{w_{\infty}}}=\sqrt{\frac{w_{c}}{w_{c, 0}}}
$$

The third Helmoltz law of vortex motion states that the change in streamwise vorticity of a vortex filament is directly proportional to the change in cross-sectional area $A_{c}{ }^{23}$, Eq. 5 . Therefore, the change in vorticity in the vortex core can be determined using Eq. 6, which applies to both the peak vorticity $\left(\omega_{z, \max } / \omega_{z, \max , 0}\right)$ and the average vorticity $\left(\omega_{z, a v} / \omega_{z, a v, 0}\right)$. 


$$
\begin{gathered}
\omega_{z} A_{c}=\omega_{z, 0} A_{c, 0} \\
\frac{\omega_{z, \max }}{\omega_{z, \max , 0}}=\frac{\omega_{z a v}}{\omega_{z a v, 0}}=\frac{w_{c}}{w_{c, 0}}=\left(\frac{r_{c, 0}}{r_{c}}\right)^{2}
\end{gathered}
$$

Finally, conservation of angular momentum dictates that $r_{c} V_{\theta, \max }$ is constant at the core radius, so the change in peak tangential velocity $\left(V_{\theta, \max } / V_{\theta, \max , 0}\right)$ is inversely proportional to the change in core radius, Eq. 7.

$$
\frac{V_{\theta}}{V_{\theta, 0}}=\sqrt{\frac{w_{c}}{w_{c, 0}}}=\frac{r_{c, 0}}{r_{c}}
$$

\subsection{Model for vorticity diffusion}

A simple model for vorticity diffusion in streamwise vortices can be obtained from solutions to the vorticity equation. For axisymmetric flow about the $z$-axis, the evolution of streamwise vorticity is given by Eq. $8^{35}$, where $v$ is the kinematic viscosity.

$$
\frac{\partial \omega_{z}}{\partial t}=v \nabla^{2} \omega_{z}
$$

A solution to Eq. 8 is given by Eq. 9, which expresses the evolution of the radial distribution of $\omega_{z}$ as a function of time $t$, where $\Gamma_{0}$ is the total circulation of the vortex. Equation 9 corresponds to the well-known Lamb-Oseen vortex solution ${ }^{35}$. The solution is for a line vortex of strength $\Gamma_{0}$ and of infinitesimal cross sectional area at $t=0 \mathrm{~s}$. From Eq. 9 it is possible to derive the corresponding solution for the tangential velocity distribution, Eq. 10.

$$
\begin{gathered}
\omega_{z}(r)=\frac{\Gamma_{0}}{4 \pi v t} e^{-r^{2} / 4 v t} \\
V_{\theta}(r)=\frac{\Gamma_{0}}{2 \pi r}\left(1-e^{-r^{2} / 4 v t}\right)
\end{gathered}
$$

The maximum of Eq. 10 corresponds to the vortex core radius, Eq. 11, where $\alpha_{l}=1.256$ is the Lamb-Oseen constant ${ }^{33}$.

$$
r_{c}(t)=\sqrt{4 \alpha_{l} v t}
$$


Squire ${ }^{28}$ proposed a modification to the Lamb-Oseen model to include the effects of turbulent diffusion through an eddy viscosity approach. By performing Reynolds averaging on the vorticity equation (Eq. 8), Squire demonstrated that the evolution of the core radius could be expressed as Eq. 12, where $\delta$ is termed the apparent diffusion coefficient and $r_{c, 0}$ is the initial core radius, typically taken to be the core radius at the trailing edge of the wing for wing-tip vortices.

$$
r_{c}=\sqrt{r_{c, 0}^{2}+4 \alpha_{l} \delta v t}
$$

Squire postulated that as vortex Reynolds number increases, so does the average influence of turbulent momentum transfer. Therefore, $\delta$ can be expressed as Eq. 13, where $a_{l}$ is an empirical constant.

$$
\delta=1+a_{1}\left(\frac{\Gamma_{0}}{v}\right)
$$

The Squire model in Eqs. 12 and 13 has been shown to be in good agreement for a wide range of wing-tip vortex measurements in uniform freestream flow ${ }^{33}$. It was demonstrated that the growth rates are approximately laminar $(\delta=1.0)$ until $\operatorname{Re}_{v} \mathrm{O}\left(10^{4}\right)$, after which $\delta$ increases in proportion to $R e_{v}$. The value of a has been found to vary between $\mathrm{O}\left(10^{-4}\right)$ to $\mathrm{O}\left(10^{-5}\right)$ for a wide range of wing-tip vortex measurements. From Ramasamy et al. ${ }^{34}, a_{1}=6.5 \times 10^{-5}$ is an appropriate assumption. Values of $\delta$ of between 3.2 and 7.7 are expected for the vortices which have been investigated in this research (Table 2). Therefore, in the unperturbed flow, the vortices of interest in this research are expected to feature diffusion rates which are somewhat larger than those attributed to laminar viscosity alone.

Although the Squire model is derived under the assumption of uniform streamwise flow, it is reasonable to assert that the model provides a suitable representation of the rate at which diffusion acts to change the vortex characteristics in contracting flows. As a result, it is proposed that the model is used to estimate the likely contribution of diffusion $\left(\Delta r_{c, D}\right)$ during intensification, with knowledge of the convection time $t$ and an appropriate value of $\delta$. Consequently, $\Delta r_{c, D}$ is evaluated using Eq. 14, where $\delta_{i}$ is the apparent diffusion coefficient for intensification, $r_{c, 0}$ is the core radius at the start of intensification, and $t$ is the convection time.

$$
\Delta r_{c, D}=\sqrt{r_{c, 0}^{2}+4 \alpha_{l} \delta_{i} v t}-r_{c, 0}
$$


The value of the apparent diffusion coefficient $(\delta)$ in uniform, freestream flow is given by the Squire vortex model in Eq. 13 for a known vortex Reynolds number. However, the value $\delta_{i}$ for flows with vortex intensification is not currently known. An expression for $\delta_{i}$ (Eq. 15) can be obtained from the insertion of Eq. 3 and Eq. 14 into Eq. 2, and rearranging for $\delta_{i}$. In the present study, the measured values of $w_{c}$ were used to estimate $r_{c, v f}$ with Eq 3. The convection time $t$ was calculated using Simpson's Rule applied to the discrete measurements of $w_{c}(z)$ at each axial location of the measurement planes, where $t=0 \mathrm{~s}$ at $z / D_{i}=2.25$.

$$
\delta_{i}=\frac{\left(r_{c, 0}+r_{c}-r_{c, V F}\right)^{2}-r_{c, 0}^{2}}{4 \alpha_{l} \delta v t}
$$

The result from Eq. 15 provides a measure of the apparent levels of diffusion over the time scale associated with the contraction. A value of $\delta_{i}$ of 0 occurs when the vortex core radius agrees with VF theory and the contribution from diffusion $\left(\Delta r_{c, D}\right)$ is zero. Consequently, it is possible to use the present experimental measurements to determine the value of $\delta_{i}$ which accounts for the differences between VF theory and the measurements.

\subsection{Model summary}

In summary, the proposed model uses Eq. 2 to determine the change in vortex core radius $\left(r_{c} / r_{c, 0}\right)$, where Eq. 3 and Eq. 14 are used to compute the inviscid $\left(\Delta r_{c, V F}\right)$ and diffusive $\left(\Delta r_{c, D}\right)$ contributions to the overall change in vortex core radius. A relationship for $\delta_{\mathrm{i}}$ is required as an input for Eq. 14 and is developed in the present study.

To determine the changes in peak tangential velocity, conservation of angular momentum is applied to relate the change in core radius to the change in peak tangential velocity (Eq. 16). Finally, Helmholtz $3^{\text {rd }}$ law is employed to determine the changes in peak and average streamwise vorticity, Eq. 17.

$$
\begin{gathered}
\frac{V_{\theta, \max }}{V_{\theta, \max , 0}}=\frac{r_{c, 0}}{r_{c}} \\
\frac{\omega_{z, \max }}{\omega_{z, \max , 0}}=\frac{\omega_{z a v}}{\omega_{z a v, 0}}=\left(\frac{r_{c, 0}}{r_{c}}\right)^{2}
\end{gathered}
$$




\section{Results}

\subsection{Assessment of apparent diffusion coefficient}

In the absence of a contracting streamtube, the flow field associated with the wing-tip vortex $\left(\operatorname{Re}_{\mathrm{v}}=1.6 \mathrm{Re}_{\mathrm{v}, \mathrm{ref}}\right)$ comprises axisymmetric distributions of tangential velocity (Error! Reference source not found.(a)) and streamwise vorticity (Error! Reference source not found.(b)). The peak tangential velocity, which is located at the core radius, corresponds to $0.61 w_{0}$. The streamwise vorticity $\left(\omega_{2} D_{i} / w_{0}\right)$ reaches a maximum of 38.8 at the centre of the vortex and is primarily contained within the core region. The preceding characteristics are in agreement with typical measurements for semi-span rectangular wings at the present angle of attack $\alpha_{\mathrm{vg}}{ }^{36}$.

The vortex core flow features high levels of turbulence intensity (Error! Reference source not found.(c)). A maximum of $T I=0.09 w_{0}$ is found at the centre of the vortex which is comparable to similar measurements ${ }^{37-40}$. This result confirms that the present measurements are within the range of vortex Reynolds numbers in which turbulent momentum transfer is expected to influence the overall diffusion rates of the vortex ${ }^{33}$.

For a typical ingestion configuration $\left(\mathrm{VR}=5.1, \mathrm{Re}_{\mathrm{v}}=1.6 \mathrm{Re}_{\mathrm{v}, \mathrm{ref}}\right)$, the vortex responds to the flow contraction and is subject to intensification, as demonstrated by the reduction in core radius and the increase in peak tangential velocity (Error! Reference source not found.(a)). At $z / D_{i}=0.2$, the core radius reduces by $46 \%$ relative to the value in the unperturbed flow, and there is a corresponding rise in peak tangential velocity of $79 \%$. Furthermore, the peak streamwise vorticity increases by 165\% (Error! Reference source not found. (b)).

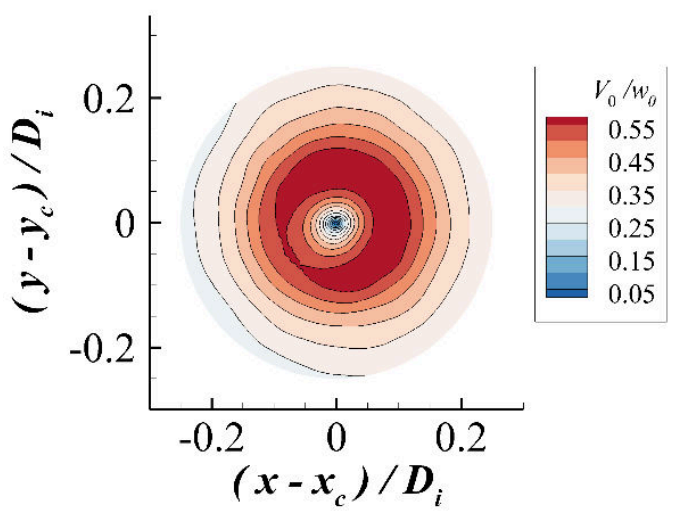

(a)

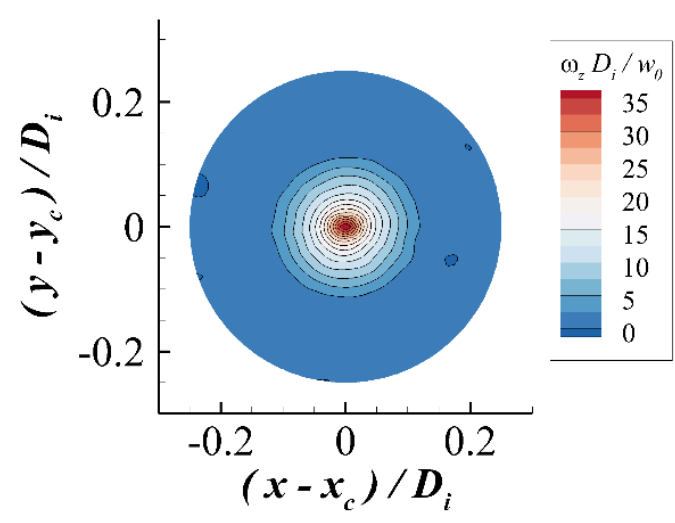

(b) 


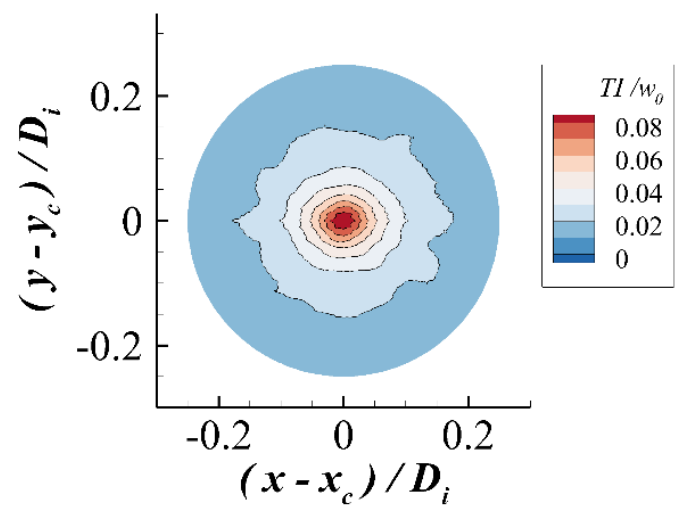

(c)

Figure 3: Flow field of wing-tip vortex in freestream flow, $\mathbf{R e}_{\mathrm{v}}=1.6 \mathbf{R e}_{\mathrm{v}, \text { ref, }}$ (a) Tangential velocity $\left(V_{\theta} / / W_{0}\right)$, (b) Streamwise vorticity $\left(\omega_{z} D_{i} / W_{0}\right)$, (c) Turbulence intensity $\left(T I / W_{0}\right)$

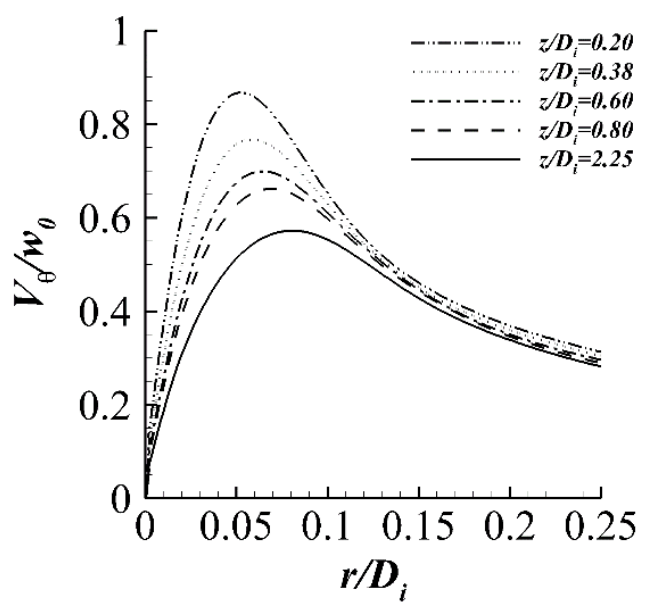

(a)

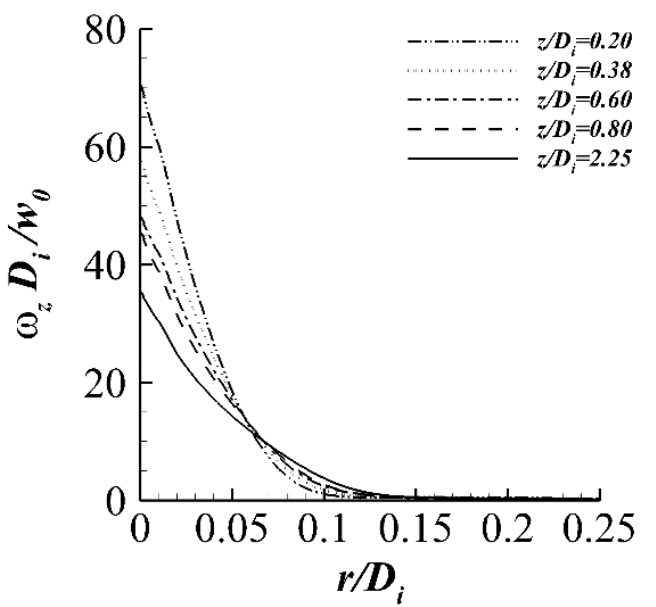

(b)

Figure 4: Circumferential-average profiles for $R e_{v}=1.6 R e_{v}$,ref, $V R=5.1$, as a function of distance from the intake highlight plane $\left(z / D_{i}\right)$, (a) Tangential velocity $\left(V_{\theta} / W_{0}\right),(\mathrm{b})$

Streamwise vorticity $\left(\omega_{z} D_{i} / w_{0}\right)$

A comparison between the measured vortex core radius $\left(r_{c} / r_{c, 0}\right)$ and VF theory, for $R e_{v}=1.6 R e_{v, r e f}\left(R e_{v, r e f}=2.4 \times 10^{4}\right)$ and $V R$ of 2.0, 5.1 and 10.3, (Error! Reference source not found.(a)), demonstrates that the vortex undergoes low levels of diffusion, and as a result the core radius is in close agreement VF theory. For example, at $w_{c} / w_{c, 0}=2.0$, the differences between VF theory and the measurements are approximately $5 \%$ for $r_{c} / r_{c, 0}$. At greater levels of flow contraction, the vortex is subject to lower levels of intensification than predicted from VF theory. At $w_{c} / w_{c, 0}=4.1$, the difference between the measurements 
and VF theory reaches $10 \%$ for $r_{c} / r_{c, 0}$, which demonstrates that vorticity diffusion becomes increasingly important as the streamtube contraction level increases. Note that the assumption of conservation of angular momentum at the core radius, as employed in VF theory (Eq. 7), is valid in the current experiments as $\Gamma_{c}$ remains constant to within $3 \%$, Error! Reference source not found.(b).

The apparent diffusion coefficient $\delta_{i}$ has been computed using Eq. 15 (Error! Reference source not found.(c)), where $w_{c} *=\left(w_{c} / w_{c, 0}-1\right)$ is the normalised streamtube contraction at the measurement plane. For $w_{c} *<1.0, \delta_{i}$ has a value of between 0 and 5 , which is of a similar order to the value which is expected for freestream vortex convection at this $R e_{v}$ ( $\delta=4.4$, Table 2 ). However, due to the short convection time scales associated with intensification, the overall effect on the vortex characteristics is small. This is illustrated in Error! Reference source not found.(a) - (c) using example solutions to the Squire model for uniform freestream flow $\left(\Delta r_{c, V F}=0\right)$ for the typical range of convection time scales $\left(t^{*}=t w_{\text {ref }} / c\right)$ as observed in the present experimental work (Table 2). For 1.0Re $e_{v, r e f}$, $V R=5.1$, the convection time scale as computed from integration of the measured axial velocity is $t^{*}=0.80$. The estimated increase in $r_{c}$ is $1.2 \%$, with a corresponding reduction of $V_{\theta}$ and $\omega_{z, \max }$ of $1.2 \%$, and $2.5 \%$, respectively (Error! Reference source not found.). Therefore, for mild but rapid streamtube contractions, the integrated effect of diffusion on the vortex is low due to the negligible the diffusion rates of the unperturbed vortex, coupled with the fact that the vortex undergoes only small changes due to intensification. Consequently VF theory provides a good description of the evolution of the vortex. For mild contractions which take place over a larger $t^{*}$, it is anticipated that diffusion effects would become more apparent which would result in a non-zero value of $\delta_{i}$ at $w_{c}^{*}=0$. 


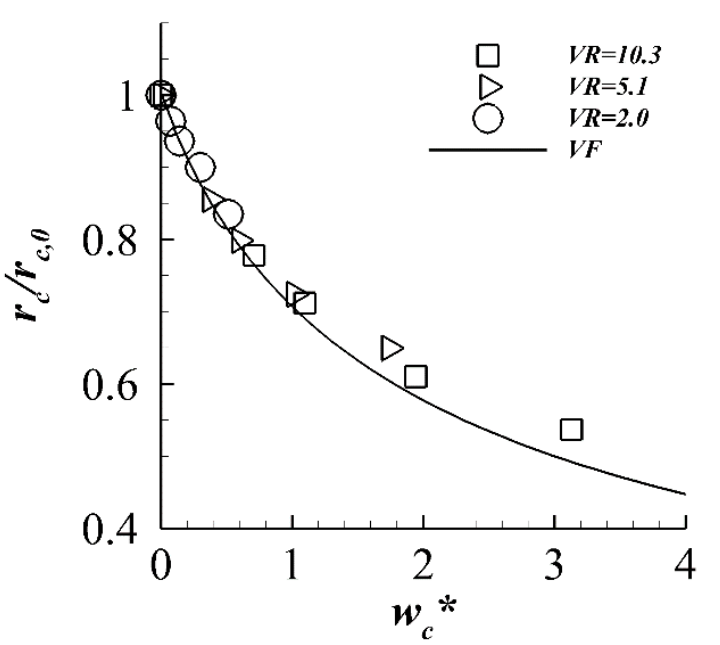

(a)

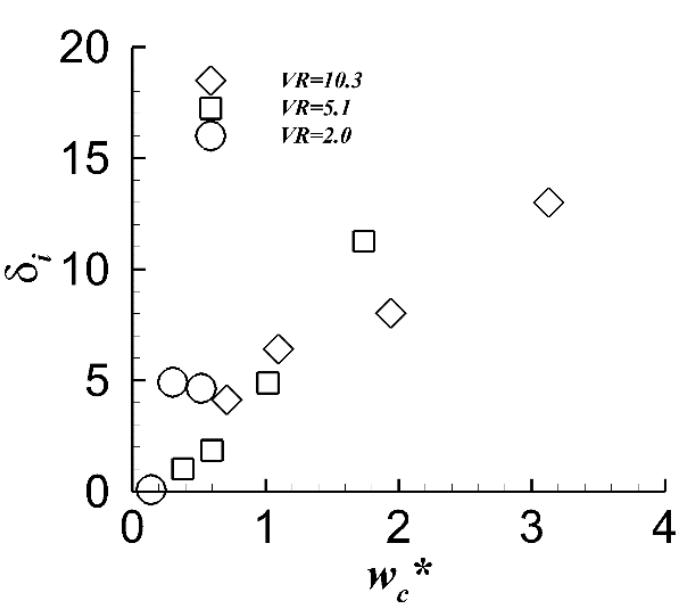

(c)

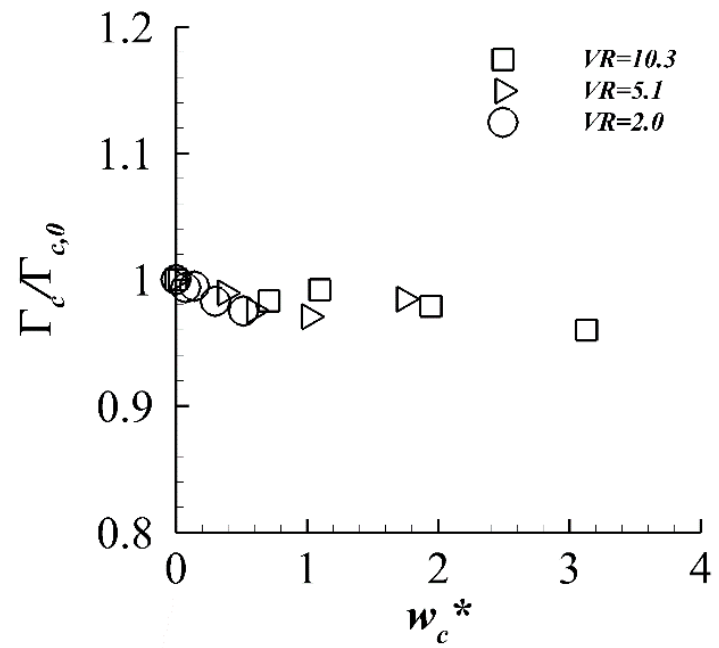

(b)

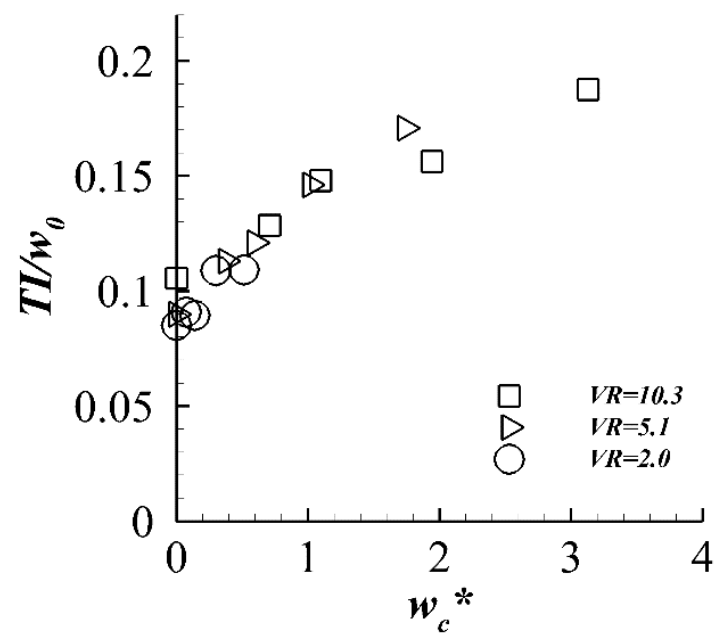

(d)

Figure 5: Data for $R e_{v}=1.6 R e_{v}$,ref, for intake velocity ratios (VR) of 10.3, 5.1 and 2.0, as a function of normalised local velocity ratio $\left(w_{c}{ }^{*}=w_{c} / w_{c, 0}-1\right)$, (a) Change in vortex core radius $\left(r_{c} / r_{c, 0}\right)$ compared to vortex filament (VF) theory, (b) Change in vortex core circulation $\left(\Gamma_{\mathrm{c}} / \Gamma_{\mathrm{c}, 0}\right)$, (c) Apparent diffusion coefficient $\left(\delta_{\mathrm{i}}\right)$, (d) Turbulence intensity $\left(T \mathrm{Tl} / \mathrm{w}_{0}\right)$

As the contraction levels grow, there is a corresponding rise in the diffusion coefficient (Error! Reference source not found.(c)). A maximum of $\delta_{i}=13.0$ is obtained at $w_{c} *=3.1$, which is three times greater than the value which is expected for the vortex in uniform flow ( $\delta=4.4$, Table 2). The gradual increase in $\delta_{i}$ as a function of $w_{c} *$ can be attributed to two factors. Firstly, as illustrated from the Squire vortex model (Error! Reference source not found.(a)-(c)), the rate at which vorticity diffusion takes place increases notably when the core radius is reduced. For example, for $\mathrm{r}_{\mathrm{c}, 0}=0.5 r_{c, \text { ref }}$ for the same vortex Reynolds number. As a result, the change in core radius is 4.7 times that for $r_{c, \text { ref }}$ for a convective time of $t^{*}=0.80$. There are changes of a similar order of magnitude 
for $V_{\theta}$ and $\omega_{z, \max }$. This increase in diffusion rates is because the flux of vorticity is driven by the gradients in streamwise vorticity ${ }^{41}$. Consequently, as the vortex core radius reduces during contraction, there is a corresponding increase in the rate of vorticity diffusion $\left(d r_{c} / d t\right)$. This causes an increase in $\delta_{i}$ for a particular $r_{c, 0}$ at the start of the contraction and a given $t^{*}$ At the greatest contraction level of $w_{c} *=5.5$ a peak $\delta_{i}$ of 25.3 is obtained for 1.0Re $e_{v, r e f}$ (Error! Reference source not found.), which is notably larger than the expected value of $\delta=3.2$ (Table 2). A second contribution to the rise in $\delta_{i}$ is indicated in the measured values of peak turbulence intensity $\left(T I / w_{0}\right)$, Error! Reference source not found.(d), which increases from 0.10 in the unperturbed condition to a maximum of 0.17 at $w_{c} *=3.2$. This rise in flow unsteadiness is likely to result in a corresponding increase in the importance of turbulent diffusion. Overall, the contraction level $w_{c} *$ is clearly a key parameter, and the conventional expression for $\delta$ as determined for unperturbed freestream flow (Eq. 13) is not sufficient for vortex intensification flows.

The Squire vortex model demonstrates that the rates of vorticity diffusion increase for greater values of $R e_{v}$ (Error! Reference source not found.). Prior comparisons with VF theory ${ }^{29}$, however, revealed only a weak dependence between $\operatorname{Re}_{\mathrm{v}}$ and changes in $r_{c}$ and $V \theta$. A more apparent trend was observed on the evolution of peak vorticity, such that the change in $\omega_{z, \max } / \omega_{z, \max , 0}$ reduces by $29 \%$ when $R e_{v}$ increases from $1.0 R e_{v, \text { ref }}$ to $3.1 R e_{v, r e f}$. Importantly, the increase in $R e_{v}$ was facilitated in the experiments using greater values of $w_{0}$, so sensitivity to $R e_{v}$ may have been masked by the fact that the convection time scale $\left(t^{*}\right)$ associated with the intensification process was not constant. For example, $t^{*}$ reduces from 0.80 at $1.0 R e_{v, \text { ref }}$ to 0.25 at $3.1 R e_{v, r e f}, V R=4.9$ (Table 2). When the typical differences in convective times are considered in the absence of intensification, the increase in diffusion rates is balanced by the reduction in convection time. For example, the increase in $r_{c}$ is $2.5 \%$ for $3.1 R e_{v}$,ref, compared to $1.2 \%$ for $1.0 R e_{v}$,ref. The calculation for $\delta_{i}$ removes the dependence on $t^{*}$ and, for a given $w_{c}{ }^{*}, \delta_{i}$ is somewhat sensitive to the vortex Reynolds number (Error! Reference source not found.). For example, at $w_{c} *$ of approximately 1.8 , the value of $\delta_{i}$ increases from 3.3 to 11.3 when the vortex Reynolds number increases from $1.0 R e_{v, \text { ref }}$ to $1.6 R e_{v, \text { ref. }}$ However, further increases in $R e_{v}$ do not produce a corresponding rise in $\delta_{i}$. The lack of a clear sensitivity may be due to the fact that the range of $R e_{v}$ investigated in this research is modest in comparison to the range of $\operatorname{Re}_{v}\left(10^{1}\right.$ to $\left.10^{5}\right)$ for which the dependence on $\operatorname{Re}_{v}$ was identified by Bhagwat et al. ${ }^{33}$. 


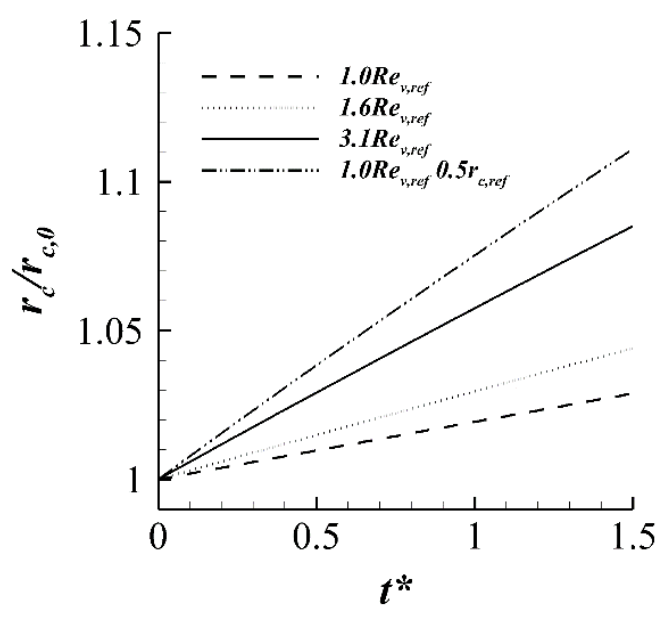

(a)

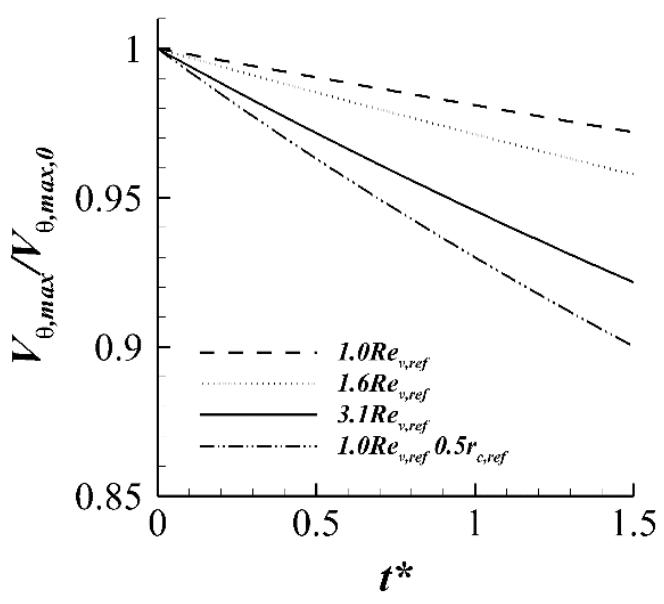

(b)

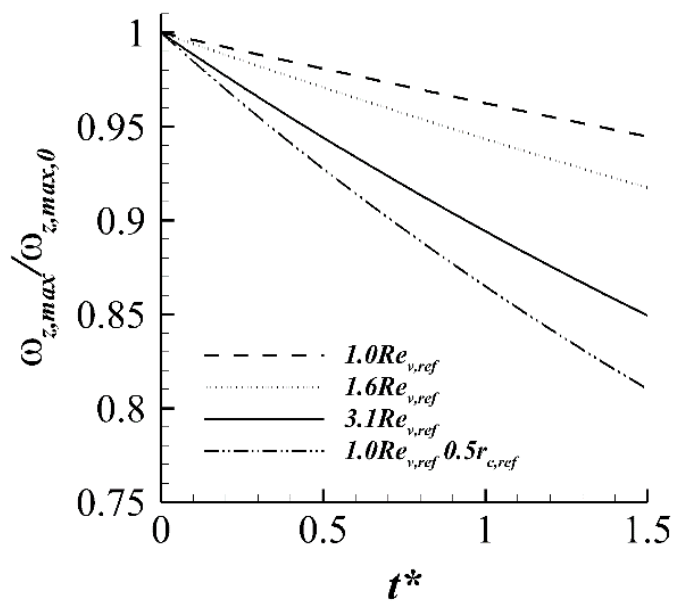

(c)

Figure 6: Solutions to the Squire vortex model as a function of convective time $\left(t^{\star}=t\right.$ $\mathbf{w}_{\text {ref }} / \mathbf{c}$ ), initial core radius, and apparent diffusion coefficient, (a) Core radius, (b) Peak tangential velocity, (c) Peak streamwise vorticity

The present calculations of $\delta_{i}$ have been compared to those which have been determined from the measurements of $V_{\theta, \max } / V_{\theta, \max , 0}$ in Garbeff at al. ${ }^{25}$, Error! Reference source not found.(a), for which $R e_{v}=0.8 R e_{v, r e f}$. Equation 4 was used to relate changes in $V_{\theta}$ to changes in $r_{c}$. For $w_{c}{ }^{*}=0.41, \delta_{i}=6.5$, which is larger than the value of $\delta=2.8$ which is expected from the Squire model. A likely contribution to this is the fact that the measurements are not corrected for vortex wandering, which is expected to reduce $V_{\theta, \max }$ and therefore increase the apparent diffusion. This highlights the benefit of using synchronous planar measurements for wing-tip vortices as it is possible to mitigate the effects of wandering. 
Ananthan et al. ${ }^{8}$ reported the axial strain rate $\varepsilon$ to which the rotor wing tip vortex was subjected, from which it was possible to determine the corresponding inviscid streamtube contraction $\left(A_{0} / A\right)$ and consequently the equivalent value of $w_{c} / w_{c, 0}$ (Eq. 18).

$$
\frac{w_{c}}{w_{c, 0}}=\frac{A_{0}}{A}=\frac{1}{1+\varepsilon}
$$

A maximum $\delta_{i}$ of 0.8 is determined at $w_{c} *=1.7$ (Error! Reference source not

found.(a)). This result is lower than the expected value of 2.7 using Eq. 13, and indicates that the diffusion levels are close to laminar. It should be noted that a value of $\delta_{i}=8.0$ was used by Ananthan et al. ${ }^{8}$ to match the experiments. However, this value was employed to provide a good agreement with the $r_{c}$ evolution for the entire wake convection process, not just the intensification process. In particular, the emphasis was to match the initial evolution of the core radius immediately downstream of the wing and prior to intensification. The differences between the calculated value of $\delta_{i}$ and the values used in Ananthan et al. ${ }^{8}$ underline the importance of establishing details of the levels of diffusion which are experienced during intensification.

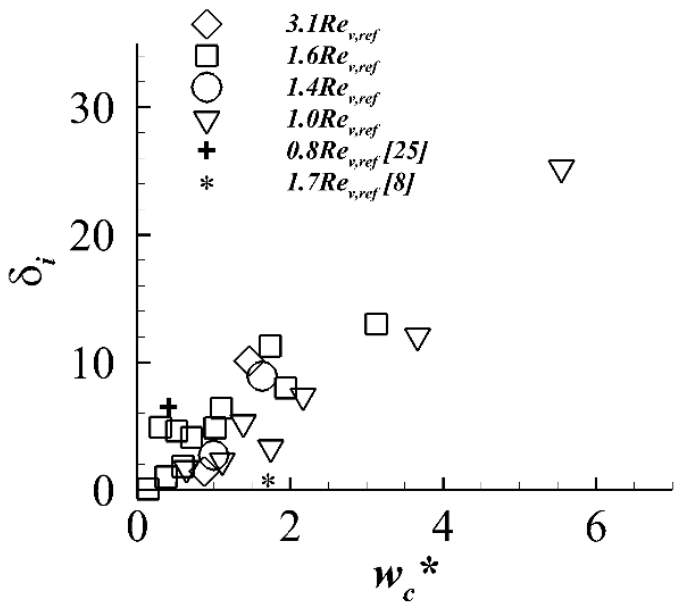

(a)

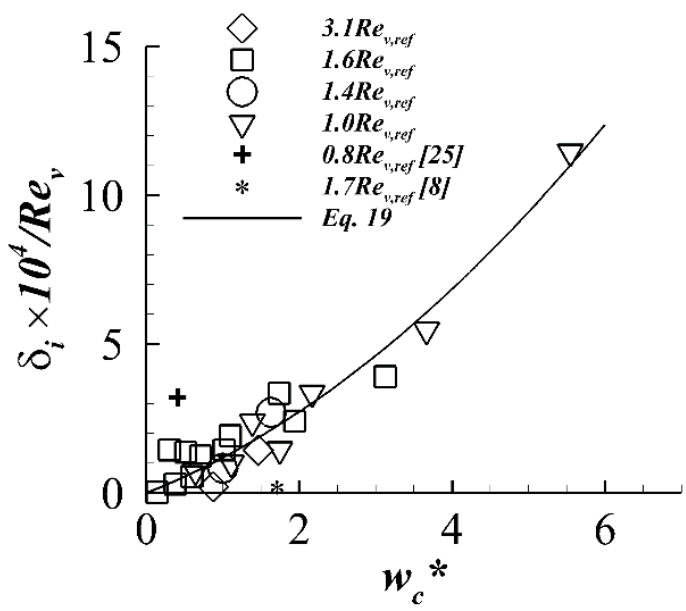

(b)

Figure 7: (a) Apparent diffusion coefficient for intensification $\left(\delta_{i}\right)$ as a function of normalised streamwise velocity ratio $w_{c}{ }^{*}$, (b) Apparent diffusion coefficient for intensification $\left(\delta_{\mathrm{i}}\right)$ normalised by vortex Reynolds number $\left(\operatorname{Re}_{\mathrm{v}}\right)$ as a function of normalised streamwise velocity ratio $w_{c}{ }^{*}$

Overall, the values of $\delta_{i}$ which have been calculated from the limited experimental data in the literature are of the same order of magnitude as those which have been determined from the present experimental measurements. The preceding analysis demonstrates that $\delta_{i}$ is primarily a function of $w_{c}{ }^{*}$, with only a weak dependence on $R e_{v}$. Results from the literature show average agreement with the trend which is observed from the present 
experimental data, although it should be emphasised that differences in the measurement methods are a likely cause. It is evident that the use of synchronous flow field measurement methods are crucial to avoid the contamination of the wing-tip vortex characteristics by wandering. Based on the present experimental measurements, it is possible to develop a suitable semi-empirical relationship for $\delta_{i}$. The dependence of $\delta_{i}$ on $R e_{v}$ can be removed in an approximate fashion by normalising by $R_{v}$ (Error! Reference source not found.(b)). As a consequence, the spread in $\delta_{i}$ at a particular $w_{c} *$ is reduced and the data can be approximated using a quadratic curve fit (Eq. 19) with a residual $\mathrm{R}^{2}$ coefficient for the curve fit of 0.94 .

$$
\delta_{i}^{*}=\frac{\delta_{i} \times 10^{4}}{R e_{v}}=0.175 w_{c}^{* 2}+1.012 w_{c}^{*}
$$

\subsection{Assessment of vortex intensification model}

Predictions have been generated using vortex filament theory (Eqs. 4, 6, 7) and the vortex intensification model (Eqs. 2, 16, 17) for $R e_{v}=1.6 R e_{v, \text { ref }}$ and $V R=2.0,5.0$ and 10.3 using the measured values of $w_{c}$. Separate results have been created for constant value of $\delta_{i}$ based the Squire model for vortices in uniform flow (Eq. 13), as well as the variable diffusion coefficient (Eq. 19).

For constant $\delta_{i}$, there is a modest improvement in the agreement between the model and the experimental measurements when compared to VF theory (Error! Reference source not found.). For $w_{c} *=3.1$, the difference in $r_{c}$ reduces from $8.3 \%$ to $5.3 \%$ (Error! Reference source not found.(a)). Similarly, differences in $V_{\theta}$ and $\omega_{z, \max }$ reduce from $13.5 \%$ to $10.1 \%$ (Error! Reference source not found.(b)), and from $55.8 \%$ to $46.6 \%$ (Error! Reference source not found.(c)), respectively. However, it is clear that the diffusion levels remain under-predicted with this approach. Notable improvements are observed with the assumption that the diffusion coefficient varies as a function of the contraction levels. For example, $r_{c}, V_{\theta}$ and $\omega_{z, a v}$ are within $3.1 \%, 1.0 \%$ and $2.1 \%$ of the experimental measurements (Error! Reference source not found.(a),(b),(d)). There remains an under-prediction in diffusion on $\omega_{z, \max }$, which is $23.2 \%$ larger than the measured value (Error! Reference source not found.(c)). Nevertheless, this represents a significant improvement in the estimation of the evolution of the vorticity terms. This result indicates that although the overall diffusion rates as observed at the core radius are captured well, the diffusion at the vortex centre is stronger than expected from the Squire vortex model.

To provide further insight into the performance of the new model, the differences between the model results and the experimental measurements have been computed for all cases (Error! Reference source not found.). For example, $r_{c, d i f f}=\left[\left(r_{c} / r_{c, 0}\right)_{\text {model }}-\right.$ 
$\left.\left(r_{c} / r_{c, 0}\right)_{\text {meas }}\right] /\left(r_{c} / r_{c, 0}\right)_{\text {meas. }}$. The inclusion of a variable $\delta_{i}$ results in a far closer agreement to the experimental measurements. At the greatest contraction level, $w_{c} *=5.5$, the differences in $r_{c} / r_{c, 0}\left(r_{c, \text { diff }}\right)$ are reduced from $27.2 \%$ to $2.5 \%$. Similarly, $V_{\theta, \text { diff }}$ reduces from $41.8 \%$ to $5.8 \%$. The greatest improvements are observed on the vorticity terms,

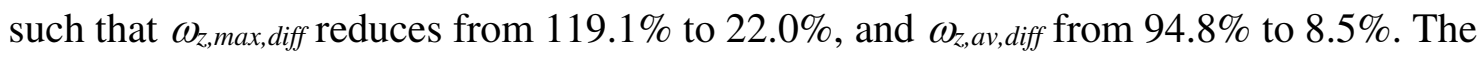
consistency between changes in $r_{c}, V_{\theta}$ and $\omega_{z, a v}$ is maintained in accordance with the fact that $\Gamma_{c}$ is conserved, as observed in the experimental results (Error! Reference source not found.(b)) and as assumed by the Squire vortex model. The results demonstrate that the vortex characteristics which are defined at the edge of the vortex core $\left(r_{c}, V_{\theta}\right.$ and $\left.\omega_{z, a v}\right)$ are captured well with the new model. This is likely because the evolution of $r_{c}, V_{\theta}$ and $\omega_{z, a v}$ broadly represent the integrated effect of diffusion across the core. The largest differences between the model and the measurements are observed in the estimates of peak vorticity. In addition, $\omega_{z, \max \text {, diff }}$ remains somewhat sensitive to $\mathrm{Re}_{\mathrm{v}}$. At $w_{c} *=1.6$, the difference in $\omega_{z, \max }$ increases from $9.9 \%$ for $1.0 R e_{v, \text { ref }}$ to $47.9 \%$ at $3.1 R e_{v, \text { ref. The }}$ behaviour of the results for peak vorticity indicate that the locally higher diffusion rates at the vortex centre are not fully captured by the assumed vorticity profiles in the Squire model. However, overall the results demonstrate that a Squire model based approach for vorticity diffusion provides a good representation of the integral parameters at the edge of the vortex core, and results in notably improved estimates for vorticity terms. 


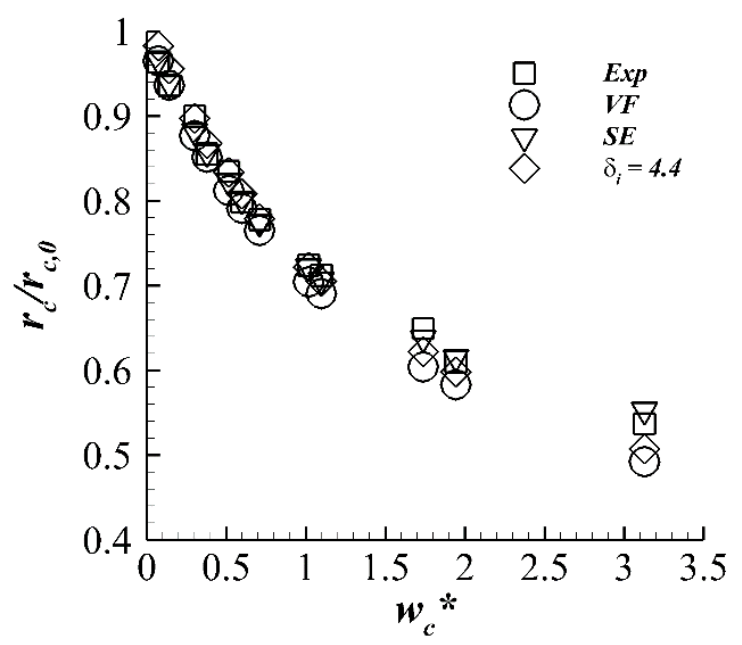

(a)

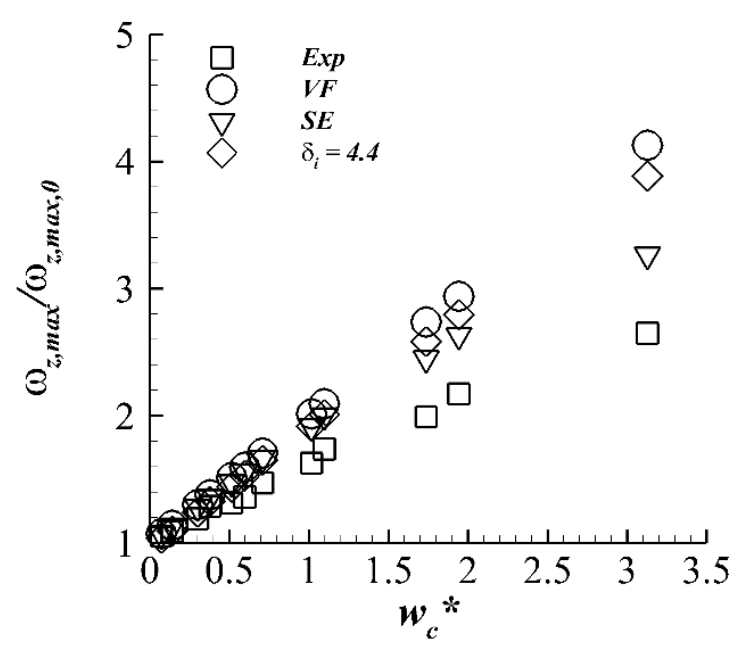

(c)

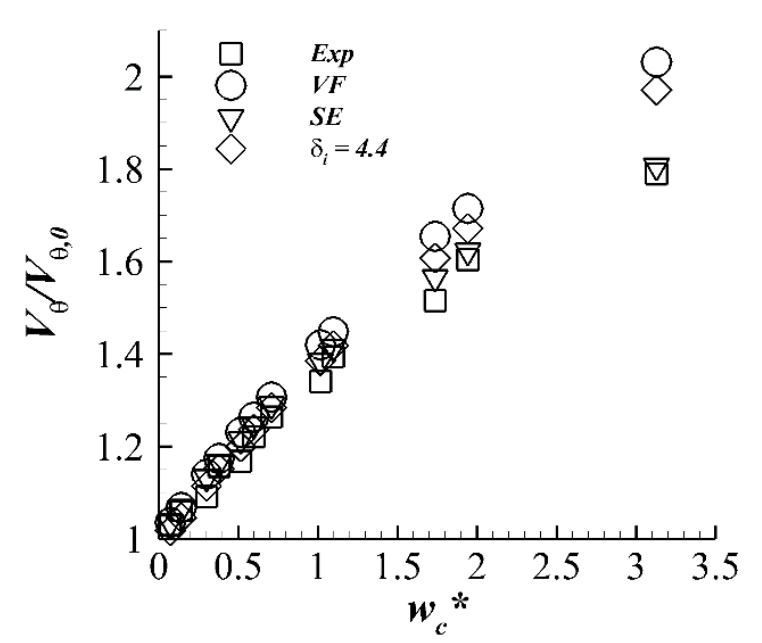

(b)

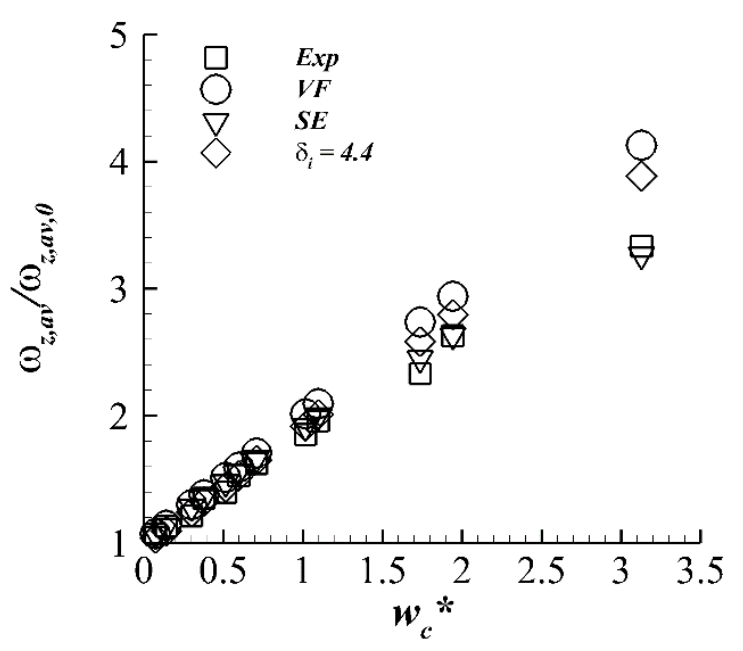

(d)

Figure 8: Comparison between experimental measurements (Exp), vortex filament theory (VF), variable $\delta_{i}$ semi-empirical model, Eq. 19 (SE) and a constant $d_{i}$ assumption $\left(\delta_{i}=4.4\right.$, Eq. 12), as a function of streamwise velocity ratio $w_{c}{ }^{*}, \operatorname{Rev}=1.6 \operatorname{Re}_{v}$,ref, (a) core radius, (b) Peak tangential velocity, (c) Peak streamwise vorticity, (d) Average core streamwise vorticity 


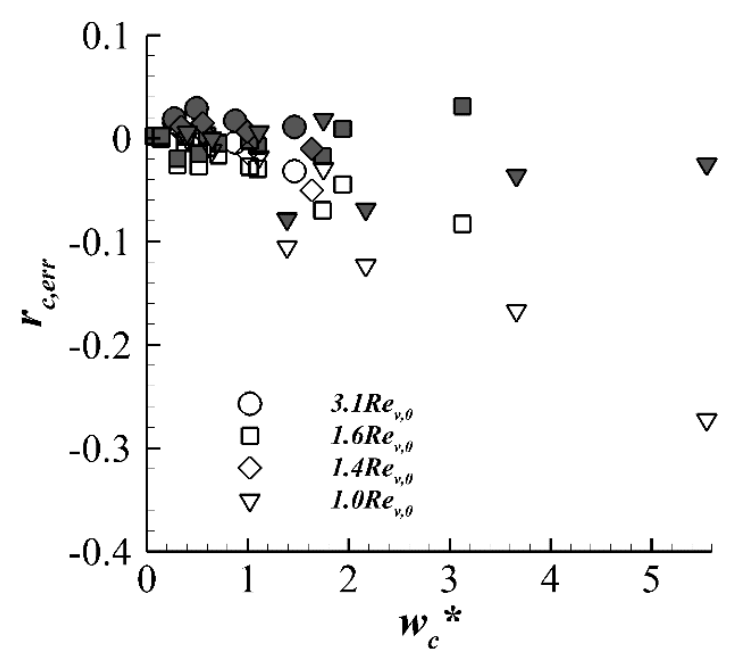

(a)

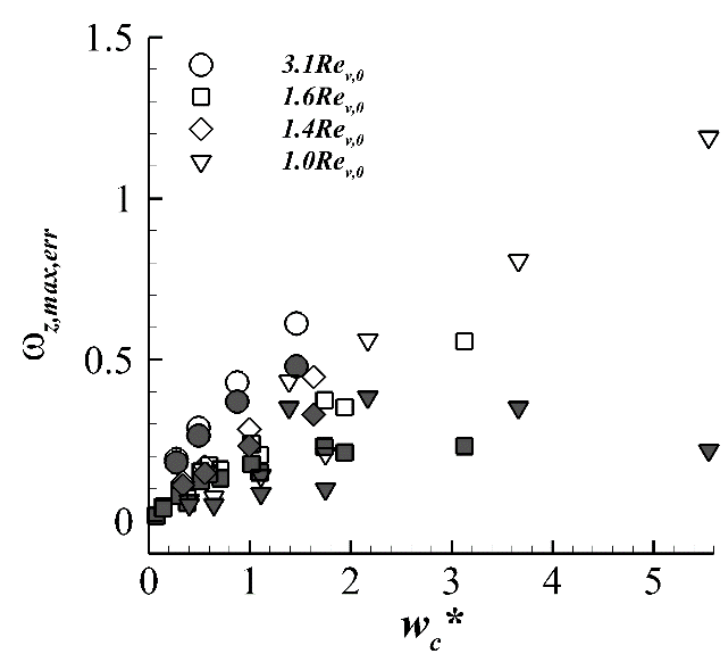

(c)

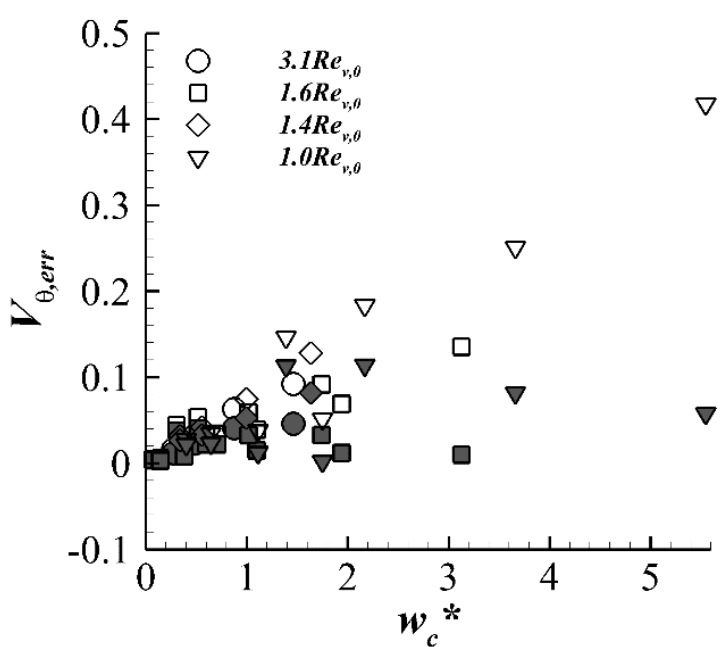

(b)

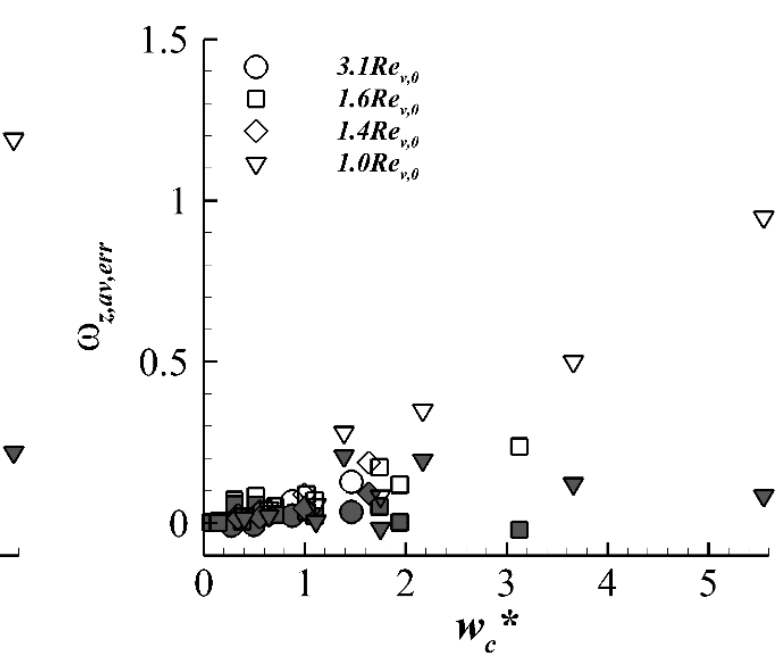

(d)

Figure 9: Difference between experimental measurements and model results, vortex filament theory (open symbols), and variable $\delta_{i}$ semi-empirical model, Eq. 19 (filled symbols), as a function of streamwise velocity ratio $w_{c}{ }^{*}$ and vortex Reynolds number, (a) Vortex core radius, (b) Peak tangential velocity, (c) Peak streamwise vorticity, (d)

\section{Average core streamwise vorticity}

\section{Conclusions}

The impact of vorticity diffusion on vortex intensification has been studied for streamwise contractions in an external streamtube. It has been found that diffusion plays an important role in the intensification process as the contraction levels increase. The contribution of diffusion has been quantified using fundamental vortex filament theory 
coupled with the Squire vortex model for diffusion. The model has been used to provide new understanding of the levels of vorticity diffusion which take place during vortex intensification. It has been found that diffusion levels are of a similar order to those experienced in freestream flow, but are a function of the local contraction levels. For mild contractions, vorticity diffusion plays a negligible role due to the short time scales associated with the intensification, coupled with the relatively slow diffusion rates of the vortex prior to intensification. As a result, the evolution of the vortex as determined from vortex filament theory is in good agreement with experimental measurements. It is hypothesised that, for mild contractions which take place over a larger time scale, the integrated effect of diffusion becomes greater, and so vortex filament theory becomes unsuitable. Vorticity diffusion becomes increasingly important as the vortex is intensified due to the corresponding rise in spatial gradients of vorticity. The experimental measurements also show that the unsteadiness of the flow in the vortex core increases during intensification, which is expected to contribute to the overall increase in vorticity diffusion due to turbulent momentum transfer.

A semi-empirical relationship has been developed to account for vorticity diffusion as a function of the streamtube contraction levels and vortex Reynolds number. When compared to fundamental vortex filament theory, the model provides an order of magnitude reduction in the estimation errors for the vortex characteristics.

The present study has developed a semi-empirical relationship for vortices of vortex Reynolds numbers of order $10^{3}$. This is recognised as the transition period above which turbulent diffusion effects become notably stronger. Therefore, future studies should extend the model to vortices with vortex Reynolds numbers of order $10^{4}$ and above. Such a validation would demonstrate the model applicability for full-scale applications, such as the ingestion of wing-tip vortices by aircraft intakes. Additional method development may be performed for cases where the strain rate is no longer axisymmetric, for example, when a vortex is ingested at a location other than at the centerline of a contracting streamtube. The validation of the method could be extended using CFD which would permit a wide range of vortex Reynolds numbers to be assessed.

\section{Funding Statement}

The lead author of this paper was partially supported by a grant from the Engineering and Physical Sciences Research Council. The authors would like to thank Rolls Royce plc for their support. 


\section{Nomenclature}

Roman symbols

$\begin{array}{lll}a_{1} & = & \text { Empirical constant for Squire vortex model } \\ A & = & \text { Vortex core area, } \mathrm{m}^{2} \\ c & = & \text { Wing chord, } \mathrm{m} \\ D_{i} & = & \text { Intake inner diameter at reference plane, } \mathrm{m} \\ r & = & \text { Radius from vortex centre, } \mathrm{m} \\ r_{c} & = & \text { Vortex core radius, } \mathrm{m} \\ \operatorname{Re}_{c} & = & \text { Reynolds number based on wing chord } \\ \operatorname{Re}_{v} & = & \text { Vortex Reynolds number }\left(\Gamma_{\mathrm{c}} / v\right) \\ T I & = & \text { Turbulence intensity, } \mathrm{ms}^{-1} \\ t & = & \text { Convective time, } \mathrm{s} \\ V_{\theta} & = & \text { Tangential velocity component, } \mathrm{ms}^{-1} \\ w & = & \text { Streamwise velocity, ms } \\ w_{0} & = & \text { Streamwise velocity in freestream flow, } \mathrm{ms}^{-1} \\ w_{c} & = & \text { Streamwise velocity at vortex centre, } \mathrm{ms}^{-1} \\ (x, y, z) & = & \text { Cartesian coordinates in reference frame, } \mathrm{m}^{-1} \\ \left(x_{c}, y_{c}\right) & = & \text { Coordinates of vortex centre, } \mathrm{m}\end{array}$

Greek symbols

$\begin{array}{lll}\alpha_{v g} & = & \text { Angle of attack of vortex generator, } \\ \alpha_{l} & = & \text { Lamb-Oseen constant, }=1.256 \\ \delta & = & \text { Apparent diffusion coefficient for Squire vortex model } \\ \delta_{i} & = & \text { Apparent diffusion coefficient for vortex intensification } \\ \varepsilon & = & \text { Strain rate, } \mathrm{s}^{-1} \\ v & = & \text { Kinematic viscosity, } \mathrm{m}^{2} \mathrm{~s}^{-1} \\ \omega_{z} & = & \text { Streamwise vorticity, } \mathrm{s}^{-1} \\ \omega_{z . a v} & = & \text { Average streamwise vorticity in vortex core }=\Gamma_{\mathrm{c}} / \pi \mathrm{r}_{\mathrm{c}}{ }^{2}, \mathrm{~s}^{-1} \\ \Gamma_{c} & = & \text { Vortex core circulation, } \mathrm{m}^{2} \mathrm{~s}^{-1} \\ \Gamma_{0} & = & \text { Vortex total circulation, } \mathrm{m}^{2} \mathrm{~s}^{-1}\end{array}$

Abbreviations $=\quad$ (Stereoscopic) Particle Image Velocimetry

$=\quad$ Laser Doppler Velocimetry

Subscripts

$\begin{array}{lll}D & = & \text { Estimate from vortex diffusion model } \\ \text { diff } & = & \text { Difference between model prediction and experimental } \\ & \text { measurement, } & \left.\left.<.\rangle_{\text {diff }}=[<.\rangle_{\text {model }}-<.\right\rangle_{\text {meas }}\right] /<.>_{\text {meas }} \\ \text { max } & = & \text { Peak value }\end{array}$


ref $=$ Reference value in unperturbed freestream flow

$V F \quad=\quad$ Estimate from vortex filament theory 


\section{References}

1. Cousins WT. History, Philosophy, Physics, and Future Directions of Aircraft Propulsion System/Inlet Integration. In: Proceedings of ASME Turbo Expo 2004 Power for Land, Sea, and Air. Vienna, Austria. Epub ahead of print 2004. DOI: 10.1115/GT2004-54210.

2. Gatlin G, Vicroy D, Carter M. Experimental Investigation of the Low-Speed Aerodynamic Characteristics of a 5.8-Percent Scale Hybrid Wing Body Configuration. 30th AIAA Appl Aerodyn Conf. Epub ahead of print 2012. DOI: 10.2514/6.2012-2669.

3. Green JS. Forced Response of a Large Civil Fan Assembly. In: ASME. Turbo Expo: Power for Land, Sea, and Air, Volume 5: Structures and Dynamics, Parts A and B. 2008, pp. 685-692.

4. Mitchell GA. Effect of inlet ingestion of a wing tip vortex on compressor face flow and turbojet stall margin, NASA TM X-3246. 1975.

5. SAE International. A Methodology for Assessing Inlet Swirl Distortion, AIR5686. 2007. Epub ahead of print 2007. DOI: https://doi.org/10.4271/AIR5686.

6. Saffman P. Vortex Dynamics. Cambridge: Cambridge University Press, 1993. Epub ahead of print 1993. DOI: 10.1017/CBO9780511624063.

7. Hall M. Vortex Breakdown. Annu Rev Fluid Mech 1972; 4: 195-217.

8. Ananthan S, Leishman JG. Role of Filament Strain in the Free Vortex Modeling of Rotor Wakes. J Am Helicopter Soc 2004; 49: 176-191.

9. Ramasamy M, Leishman JG. Interdependence of diffusion and straining of helicopter blade tip vortices. J Aircr 2004; 41: 1014-1024.

10. Karpatne A, Sirohi J, Mula S, et al. Vortex Ring Model of Tip Vortex Aperiodicity in a Hovering Helicopter Rotor. J Fluids Eng 2014; 136: 071104.

11. Yang Y, Veldhuis LLM, Eitelberg G. Aerodynamic impact of a streamwise vortex on a propeller. Aerosp Sci Technol 2017; 70: 108-120.

12. Suerich-Gulick F, Gaskin SJ, Villeneuve M, et al. Free surface intake vortices: Theoretical model and measurements. J Hydraul Res 2014; 52: 502-512. 
13. Qureshi I, Povey T. A combustor-representative swirl simulator for a transonic turbine research facility. Proc Inst Mech Eng Part G J Aerosp Eng 2011; 225: $737-748$.

14. Qureshi I, Smith AD, Chana KS, et al. Effect of Aggressive Inlet Swirl on Heat Transfer and Aerodynamics in an Unshrouded Transonic HP Turbine. $J$ Turbomach 2012; 134: 1-13.

15. Chaluvadi, V. S. P.; Kalfas, A. I.; Banieghbal, M. R.; Hodson, H. P.; Denton JD. Blade-Row Interaction in a High-Pressure Turbine. J Propuls Power 2001; 17: 892-901.

16. Poehler, Thorsten; Niewoehner, J.; Jeschke, P.; Guendogdu Y. Airfoil Design in a 1.5 Stage Axial Turbine Part I : Design and Novel Numerical Analysis Method. In: Proceedings of ASME Turbo Expo 2014: Turbine Technical Conference and Exposition. Düsseldorf, Germany, 2014.

17. Prandtl L. Attaining a Steady Air Stream in Wind Tunnels. Tech Memo Natl Advis Comm Aeronaut 1933; 40.

18. Batchelor GK. An Introduction to Fluid Dynamics. Cambridge University Press, 1967.

19. Buntine JD, Saffman PG. Inviscid Swirling Flows and Vortex Breakdown. Proc $R$ Soc A Math Phys Eng Sci 1995; 449: 139-153.

20. Rusak Z, Meder CC. Near-Critical Swirling Flow in a Slightly Contracting Pipe. AIAA J 2004; 42: 2284--2284.

21. Leclaire, B.; Jacquin, L.; Sipp D. Effects of a contraction on a uniformly rotating flow. In: 4th AIAA Theoretical Fluid Mechanics Meeting. Toronto, Ontario, 2005.

22. Rusak, Z.; Bourquard, N.; Wang S. Vortex Breakdown in Swirling Flows in Diverging or Contracting Pipes. In: AIAA Aerospace Sciences Meeting including the New Horizons Forum and Aerospace Exposition. Grapevine, Texas, 2013.

23. Wu JZ, Ma HY, Zhou M De. Vorticity and vortex dynamics. Springer-Verlag Berlin Heidelberg, 2006. Epub ahead of print 2006. DOI: 10.1007/978-3-54029028-5.

24. Rott N. On the viscous core of a line vortex. Zeitschrift fur Angew Math 1958; 9b: 543-553. 
25. Garbeff II, T.; Huthmacher, R.; Tso, J.; Martin, P; Tung C. An Experimental Survey of a Wing-tip Vortex in a Contracted Flow. In: 40th Fluid Dynamics Conference and Exhibit. Chicago, Illinois, 2010.

26. Devenport WJ, Rife MC, Liapis SI, et al. The structure and development of a wing-tip vortex. J Fluid Mech 1996; 312: 67.

27. Iungo G V. Wandering of a Wing-Tip Vortex: Rapid Scanning and Correction of Fixed-Point Measurements. J Aircr 2017; 54: 1179-1190.

28. Squire H. The Growth of a Vortex in Turbulent Flow. Aeronaut $Q$ 1965; 16: $302-$ 306.

29. McLelland G, MacManus D, Sheaf C. The Effect of Streamtube Contraction on the Characteristics of a Streamwise Vortex. J FLUIDS Eng ASME; 137. Epub ahead of print 2015. DOI: 10.1115/1.4029661.

30. Westerweel J, Scarano F. Universal outlier detection for PIV data. Exp Fluids 2005; 39: 1096-1100.

31. Burley CL, Brooks TF, Rozier KY, et al. Rotor wake vortex definition-evaluation of 3-C PIV results of the HART-II study. In: 28th European Rotorcraft Forum, Bristol, UK. 2002.

32. Raffel M, Willert CE, Wereley ST, et al. Particle Image Velocimetry: A Practical Guide. 2007.

33. Bhagwat MJ, Leishman JG. Generalized Viscous Vortex Model for Application to Free-Vortex Wake and Aeroacoustic Calculations. In: Annual Forum ProceedingsAmerican Helicopter Society. 2008, pp. 2042-2057.

34. Ramasamy M, Leishman JG. A Reynolds Number - Based Blade Tip Vortex Model. 61 st Annu Forum Technol Disp Am Helicopter Soc 2007; 214-223.

35. Lamb SH. Hydrodynamics. 6th ed. Cambridge University Press, 1932.

36. Lee T, Pereira J. Nature of Wakelike and Jetlike Axial Tip Vortex Measurements. J Aircr 2010; 47: 1946-1954.

37. Chow JS, Zilliac GG, Bradshaw P. Mean and Turbulence Measurements in the Near Field of a Wingtip Vortex. AIAA J 1997; 35: 1561-1567. 
38. Bandyopadhyay PR, Ash RL, Stead DJ. Organized nature of a turbulent trailing vortex. AIAA J 1991; 29: 1627-1633.

39. Han YO, Leishman JG, Coyne AJ. Measurements of the Velocity and Turbulence Structure of a Rotor Tip Vortex. AIAA J 1997; 35: 477-485.

40. Phillips WR, Graham JA. Reynolds-stress measurements in a turbulent trailing vortex. J Fluid Mech 1984; 147: 353-371.

41. Crank J. The Mathematics of Diffusion. Oxford University Press, 1975. 
2019-04-01

\title{
A semi-empirical model for streamwise vortex intensification
}

\author{
McLelland, Grant
}

Sage

McLelland G, MacManus D, Sheaf C. (2019) A semi-empirical model for streamwise vortex intensification. Proceedings of the Institution of Mechanical Engineers, Part G: Journal of Aerospace Engineering, Journal of Aerospace Engineering, Volume 233, Issue 12, 2019, pp. 4396-4409 https://doi.org/10.1177/0954410019838421

Downloaded from Cranfield Library Services E-Repository 